\title{
AN EMBEDDING THEOREM FOR GROUPS
}

\author{
by C. G. CHEHATA
}

(Received 13 August, 1959)

1. Introduction. Let $G$ by any given group. A homomorphic mapping $\mu$ of a subgroup $A$ of $G$ onto a second subgroup $B$ of $G$, where $A$ and $B$ need not be distinct, is called a partial endomorphism of $G$. When $\mu$ is defined on the whole of $G$, that is when $A=G$, we call $\mu$ a total endomorphism of $G$; or simply an endomorphism of $G$.

A partial (or total) endomorphism $\mu^{*}$ of a supergroup $G^{*}$ of $G$ is said to extend (or continue) $\mu$ if $\mu^{*}$ is defined on a supergroup $A^{*}$ of $A$, that is, $\mu^{*}$ is defined for at least the elements for which $\mu$ is defined, and moreover $\mu^{*}$ coincides with $\mu$ on $A$.

In [2] B. H. Neumann and Hanna Neumann derived necessary and sufficient conditions for a partial endomorphism of a group to be extendable to a total endomorphism of a supergroup. These conditions were then generalised by the author [1], to give necessary and sufficient conditions for the simultaneous extension of two partial endomorphisms to total endomorphisms of one and the same supergroup. The conditions there obtained were then generalised using transfinite induction to any well-ordered set of partial endomorphisms.

In this paper, I derive the necessary and sufficient conditions that a partial endomorphism $\mu$ of a group $G$ can be extended to a total endomorphism $\mu^{*}$ of a supergroup $G^{*}$, with the condition that $\mu^{*}$ acts as an isomorphism on $G^{*}\left(\mu^{*}\right)^{m}$, for some given positive $m$, imposed on $\mu^{*}$.

2. Necessary conditions. Let $\mu$ be a partial endomorphism of $G$ mapping the subgroup $A \subseteq G$ onto the subgroup $B \subseteq G$.

To obtain necessary conditions for $\mu$ to be extendable to a total endomorphism $\mu^{*}$ of a supergroup $G^{*} \supseteq G$ such that $\mu^{*}$ is an isomorphism on $G\left(\mu^{*}\right)^{m}$, where $m$ is a positive integer we assume that the extension is already established.

Denote the kernel of $\mu$ by $K$ and that of $\left(\mu^{*}\right)^{n}$ by $K\left(\mu^{* n}\right)$. The canonic mapping of $G^{*}$ onto $G^{*} / K\left(\mu^{*}\right)$ induces the canonic mapping of $A$ onto $A / K$; but it also induces the canonic mapping of $A$ onto $A /\left(K\left(\mu^{*}\right) \cap A\right)$; thus

$$
K=K\left(\mu^{*}\right) \cap A=\text { kernel of } \mu .
$$

If we put

$$
L_{i}=K\left(\mu^{* i}\right) \cap G,
$$

then, as in [1] or [2], we can prove that $K\left(\mu^{* i}\right)$ are normal subgroups of $G$ such that

$$
K\left(\mu^{* i}\right) \subseteq K\left(\mu^{* j}\right) \text { for } i \leqslant j,
$$

and thus $L_{i}$ are also normal subgroups for $G$ for which

$$
L_{1} \subseteq L_{2} \subseteq \ldots
$$

and

$L_{1} \cap A$ is the kernel of $\mu$,

$$
\left(L_{i+1} \cap A\right) \mu=L_{i} \cap B \quad(i=1,2, \ldots) .
$$

Now we have

$$
K\left(\mu^{* m}\right) \subseteq K\left(\mu^{* m+1}\right)
$$


If $x \in K\left(\mu^{* m+1}\right)$, then

$$
x \mu^{* m+1}=1, \text { or }\left(x \mu^{* m}\right) \mu^{*}=1,
$$

where 1 denotes the unit element of $G^{*}$; since $\mu^{*}$ is an isomorphism on $G\left(\mu^{*}\right)^{m}$, then

i.e.

$$
x \mu^{* m}=1 \text {, }
$$

thus

$$
K\left(\mu^{* m+1}\right) \subseteq K\left(\mu^{* m}\right)
$$

(I) and (II) together give

$$
K\left(\mu^{* m}\right)=K\left(\mu^{* m+1}\right)
$$

from which follows that

Similarly we can show that

$$
\begin{aligned}
K\left(\mu^{* m}\right) \cap G & =K\left(\mu^{* m+1}\right) \cap G \\
L_{m} & =L_{m+1}
\end{aligned}
$$

Thus we have the following theorem :

$$
L_{m}=L_{m+1}=L_{m+2}=\ldots
$$

Theorem 1. For the partial endomorphism $\mu$ of the group $G$ which maps $A$ onto $B, A$ and $B$ being subgroups of $G$, to be extendable to a total endomorphism $\mu^{*}$ of a supergroup $G^{*} \supseteq G$ such that $\mu^{*}$ is an isomorphism on $G\left(\mu^{*}\right)^{m}$, it is necessary that there exists in $G$ a sequence of normal subgroups

such that

$$
L_{1} \subseteq L_{2} \subseteq \ldots \subseteq L_{m}=L_{m+1}=\ldots
$$

$$
L_{1} \cap A \text { is the kernel of } \mu \text {, }
$$

$$
\left(L_{i+1} \cap A\right) \mu=L_{i} \cap B \quad(i=1,2, \ldots, m) .
$$

From Theorem 1 follow immediately the following two corollaries.

Corollary 1. If $x \mu^{i}$ is defined, then $x \mu^{i}=1$ if and only if $x \in L_{i} \cap A$.

Proof. If $x \in L_{i} \cap A$, then

$$
x \mu \in\left(L_{i} \cap A\right) \mu=L_{i+1} \cap B,
$$

by relation (3); and since $x \mu^{2}$ is defined, then

and

$$
\begin{aligned}
& x \mu \in L_{i-1} \cap B \cap A \subseteq L_{i-1} \cap A, \\
& x \mu^{2} \in\left(L_{i-1} \cap A\right) \mu=L_{i-2} \cap B .
\end{aligned}
$$

Applying this a finite number of times, we get

$$
x \mu^{i-1} \in L_{1} \cap A,
$$

from which, because of relation (2), it follows that

$$
x \mu^{i}=1 .
$$

Conversely, if $x \mu^{i}=1$, then $x \mu^{i-1} \in L_{1} \cap A$.

But also

$$
\begin{aligned}
& x \mu^{i-1} \in B ; \\
& x \mu^{i-1} \in L_{1} \cap A \cap B \subseteq L_{1} \cap B=\left(L_{2} \cap A\right) \mu,
\end{aligned}
$$$$
\text { thus }
$$ 
i.e.

$$
x \mu^{i-2} \in L_{2} \cap A \text {. }
$$

The result follows by induction.

Corollary 2. $†$ If $x \mu^{m+1}$ is defined, then

$$
x \mu^{m+1}=1 \text { implies that } x \mu^{m}=1 .
$$

Proof: $x \mu^{m+1}=1$ implies, by Corollary 1, that

and thus

$$
\begin{aligned}
x \in L_{m+1} \cap A & =L_{m} \cap A, \\
x \mu^{m} & =1 .
\end{aligned}
$$

3. Sufficient conditions. To prove that the conditions of Theorem 1 are also sufficient, we assume that the conditions there stated are satisfied and put

Then $M$ contains a subgroup

$$
H=G / L_{1} \text {. }
$$

$$
B^{\prime}=\left(A \cup L_{1}\right) / L_{1} \sim A /\left(A \cap L_{1}\right) \sim B .
$$

An isomorphism between $B^{\prime}$ and $B$ is defined by letting $a L_{1} \in B^{\prime}$ correspond to $a \mu \in B$, where $a \in A$. We then define the group $G_{1}$ to be the free product of $G$ and $H$, amalgamating $B$ and $B^{\prime}$ according to the above isomorphism :

$$
G_{1}=\left\{G * H ; B=\left(A \cup L_{1}\right) / L_{1}\right\} ;
$$

and we let $\mu_{1}$ be the canonic mapping of $G$ onto $H$. The mapping $\mu_{1}$ extends $\mu$.

We then define in $G_{1}$ the normal groups

$$
M_{i}=\left(\left(L_{i+1} \mu_{1}\right) \cup L_{i}\right)^{G_{1}}
$$

where $X^{Y}$ denotes the normal closure of $X$ in $Y$. Then, if $i \leqslant j$,

$$
M_{i}=\left(\left(L_{i+1} \mu_{1}\right) \cup L_{\imath}\right)^{G_{1}} \subseteq\left(\left(L_{j+1} \mu_{1}\right) \cup L_{j}\right)^{G_{1}}=M_{j},
$$

since $L_{i} \subseteq L_{j}, L_{i+1} \subseteq L_{j+1}$, by relations (1). Moreover,

$$
M_{m+1}=\left(\left(L_{m+2} \mu_{1}\right) \cup L_{m+1}\right)^{\sigma_{1}}=\left(\left(L_{m+1} \mu_{1}\right) \cup L_{m}\right)^{\sigma_{1}}=M_{m},
$$

since $L_{m}=L_{m+1}=L_{m+2}$, by relations (1). Thus

$$
M_{1} \subseteq M_{2} \subseteq \ldots \subseteq M_{m}=M_{m+1}=\ldots ;
$$

i.e., in $G_{1}$ the normal subgroups $M_{i}$ satisfy (1) when the $\left\{L_{i}\right\}$ are replaced by $\left\{M_{i}\right\}$.

The proof that conditions (2) and (3) are satisfied when $M_{i}, G, H, \mu_{1}$ replace $L_{i}, A, B, \mu$ respectively, is the same as in [1] or [2]. Thus

$$
\begin{aligned}
M_{1} \cap G & \text { is the kernel of } \mu_{1}, \\
\left(M_{i+1} \cap G\right) \mu_{1} & =M_{i} \cap H \quad(i=1,2, \ldots, m) .
\end{aligned}
$$

Conditions (5), (6) and (7) give, as in Corollary 2 , the following

CoRollary 3. In $G_{1}$, if $x \mu_{1}^{m+1}$ is defined, then $x \mu_{1}^{m+1}=1$ implies that $x \mu_{1}^{m}=1$.

Thus we can repeat the process, embedding $G_{1}$ in $G_{2}$, in which a partial endomorphism $\mu_{2}$ extends $\mu_{1}$ with the conditions corresponding to (l)-(3) satisfied, and hence the condition corresponding to (4) also holds.

† I am indebted to Dr B. H. Neumann for drawing my attention to the fact that relation (4) follows from the remaining conditions. 
Then we carry on inductively.

Finally we form

$$
G^{*}=\bigcup_{n=0}^{\infty} G_{n}
$$

with $G_{0}=G$.

Define the mapping $\mu^{*}$ of $G^{*}$ as follows : For any $g \in G^{*}, g \in G_{n}$ for some suitable $n$, and we put

$$
g \mu^{*}=g \mu_{n}
$$

Thus $\mu^{*}$ defines a total endomorphism of $G^{*}$ which extends $\mu$. Also if $g \in G^{*}$ and $g \mu^{* m+1}=1$, then

$$
g \mu_{n}^{m+1}=1, \text { for some suitable } n,
$$

which implies, because of corollaries corresponding to Corollaries 2 and 3 , that

$$
g \mu_{n}^{m}=1 \text {. }
$$

Thus $\mu^{*}$ is an isomorphism on $G\left(\mu^{*}\right)^{m}$, and we get

Theorem 2. Conditions (1)-(3) of Theorem 1 are also sufficient for the partial endomorphism $\mu$ of the group $G$ to be extendable to a total endomorphism of a supergroup $G^{*} \supseteq G$ such that $\mu^{*}$ is an isomorphism on $G\left(\mu^{*}\right)^{m}$.

\section{REFERENCES}

1. C. G. Chehata, Simultaneous extension of partial endomorphisms of groups, Proc. Glasgow Math. Assoc., 2 (1954), 37-46.

2. B. H. Neumann and Hanna Neumann, Extending partial endomorphisms of groups, Proc. London Math. Soc. (3) 2 (1952), 337-348.

Faculty of Science

The UnTVERsitry

ALEXANDRLa, Eaypt 